\title{
COMBINING HIGH SPATIAL RESOLUTION OPTICAL AND LIDAR DATA FOR OBJECT-BASED IMAGE CLASSIFICATION
}

\author{
Ruonan Li ${ }^{1}$, Tianyi Zhang ${ }^{1}$, Ruozheng Geng ${ }^{1}$, Leiguang Wang ${ }^{2}$, \\ ${ }^{1}$ School of Forestry, Southwest Forestry University, Kunming, 650224, China \\ ${ }^{2}$ Institutes of Big Data and Artificial Intelligence, Southwest Forestry University, Kunming, 650024, China
}

KEY WORDS: High Spatial Resolution, Lidar, Object-Based Classification, Aerial Image, Image segmentation

\begin{abstract}
ABATRACT: In order to classify high spatial resolution images more accurately, in this research, a hierarchical rule-based objectbased classification framework was developed based on a high-resolution image with airborne Light Detection and Ranging (LiDAR) data. The eCognition ${ }^{\circledR}$ software is employed to conduct the whole process. In detail, firstly, the FBSP optimizer (Fuzzy-based Segmentation Parameter) is used to obtain the optimal scale parameters for different land cover types. Then, using the segmented regions as basic units, the classification rules for various land cover types are established according to the spectral, morphological and texture features extracted from the optical images, and the height feature from LiDAR respectively. Thirdly, the object classification results are evaluated by using the confusion matrix, overall accuracy and Kappa coefficients. As a result, a method using the combination of an aerial image and the airborne Lidar data shows higher accuracy.
\end{abstract}

\section{INTRODUCTION}

The continuous improvement of remote sensing images in spatial resolution brings both opportunities and challenges to image interpretation. More detailed structures and textures of land objects can be observed from the high spatial resolution images, meanwhile, the variation in a given land class become strong. As traditional pixel-based classification methods cannot effectively utilize the rich information contained in high resolution images, often suffer from the "pepper and salt" problem. Meanwhile, the optical remote sensing images can only capture the horizontal information of land surface but cannot explore the vertical structure of objects. Therefore, the optical remote sensing images will fail to differentiate two land classes composed of the same material. In this context, we combine optical remote sensing images and Lidar data and present a novel object-based image analysis method. As pixel-based image analysis employs every single pixel as processing unit, it cannot fully investigate the spatial information.

Meanwhile, object-based image analysis (OBIA) is based on information from a set of neighbouring pixels sharing similar spectral or spatial properties. Object-based image analysis can classify by using their spatial, semantic, textural or topological relationships with adjacent or nearby objects and can eliminate the "pepper salt" effect, resulting in the improvement of the classification accuracy.

High-resolution imagery and high-density LiDAR data provide complementary horizontal and vertical information (Tong et al., 2012, Zhou et al., 2015). For example, it is generally difficult to distinguish an objects type having different classification but the same material solely on the spectral information contained from high-resolution image, such as roads and bituminous roofs. Therefore, this paper proposes a method that solves this problem by using LiDAR data and high-resolution image. It has been demonstrated that the synergetic use of LiDAR and high-solution imagery greatly improves classification accuracy(Zhou et al.,
2015). The primary task of this paper was to build an objectbased classification rule hierarchy system using the LiDAR data and high spatial resolution optical to classify a residential area comprised of independent buildings, grass, trees, garden and road. The second purpose was to assess the general applicability and transferability of the rule hierarchy system to different areas(Salehi et al., 2012). The eCognition ${ }^{\circledR}$ software is employed to conduct the whole process. The hierarchical rule-based objectbased classification framework was developed using optical and LiDAR image according to the spectral, morphological and texture features. To assess the transferability of the developed rule-set to different areas, the similar rule-set was used to a different area of the optical and LiDAR image(Salehi et al., 2012).

\section{DATASETS AND STUDY AREAS}

Two different parts of the city of Vaihingen in German were chosen as the study areas in this research. The data set is a subset of the data used for the test of digital aerial cameras carried out by the German Association of Photogrammetry and Remote Sensing. One group of the data source is the digital aerial images with $8 \mathrm{~cm}$ ground resolution which consists of 3 bands including Near Infrared (NIR), Red and Green bands. The other one is digital surface mode (DSM) data with a ground resolution of 9 $\mathrm{cm}$, which was derived from a Lidar. Two sets of data covering the different area are used in this study. The Data 1(Figure (a)) is a purely residential area including obvious roads, independent houses, grassland and trees (http://www2.isprs.org/commissions/comm3/wg4/detectionand-reconstruction.html).

The scene of Data 2(Figure (c)) is located in the central area of Vaihingen city, which is characterized by the complex shape of the buildings that surrounded by trees. The Data 1 was used for developing the rule-set, and the Data 2 was used for assessing the availability of the developed classification framework. 


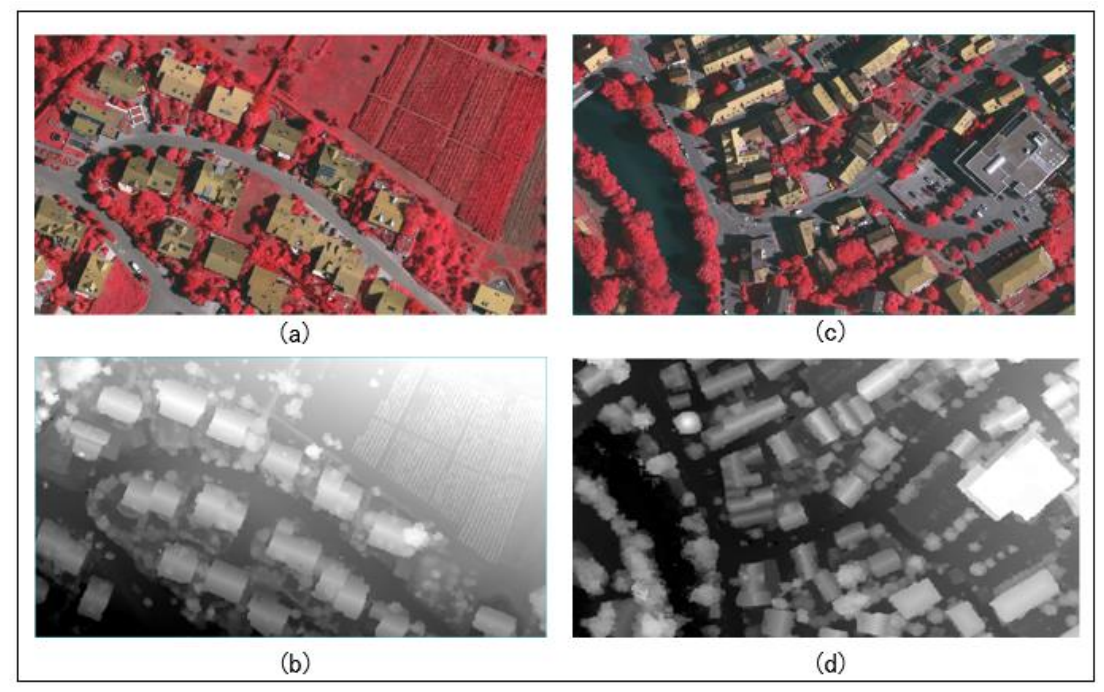

Figure 1. Datasets: (a) aerial image (Data 1), (b) DSM (Data 1), (c) aerial image (Data 2), (d) DSM (Data 2)

\section{METHODOLOGY}

The whole methodology is showed in Figure 2. In the first step, the nDSM data is derived from DSM. Then, the aerial image is segmented into three hierarchical levels from bottom to up. Thirdly, the hierarchical rule-based classification is conducted. During the process, the Normalized Difference Vegetation Index
(NDVI) feature is firstly used to divide the whole image into vegetation and non-vegetation. Vegetation areas were classified to trees, grass and garden using height and texture information. Non-vegetation areas are further classified to buildings, roads and shadows using spectral information such as brightness and NDVI, and morphological features such as shape, area and rectangular fit, and relationships between different levels.

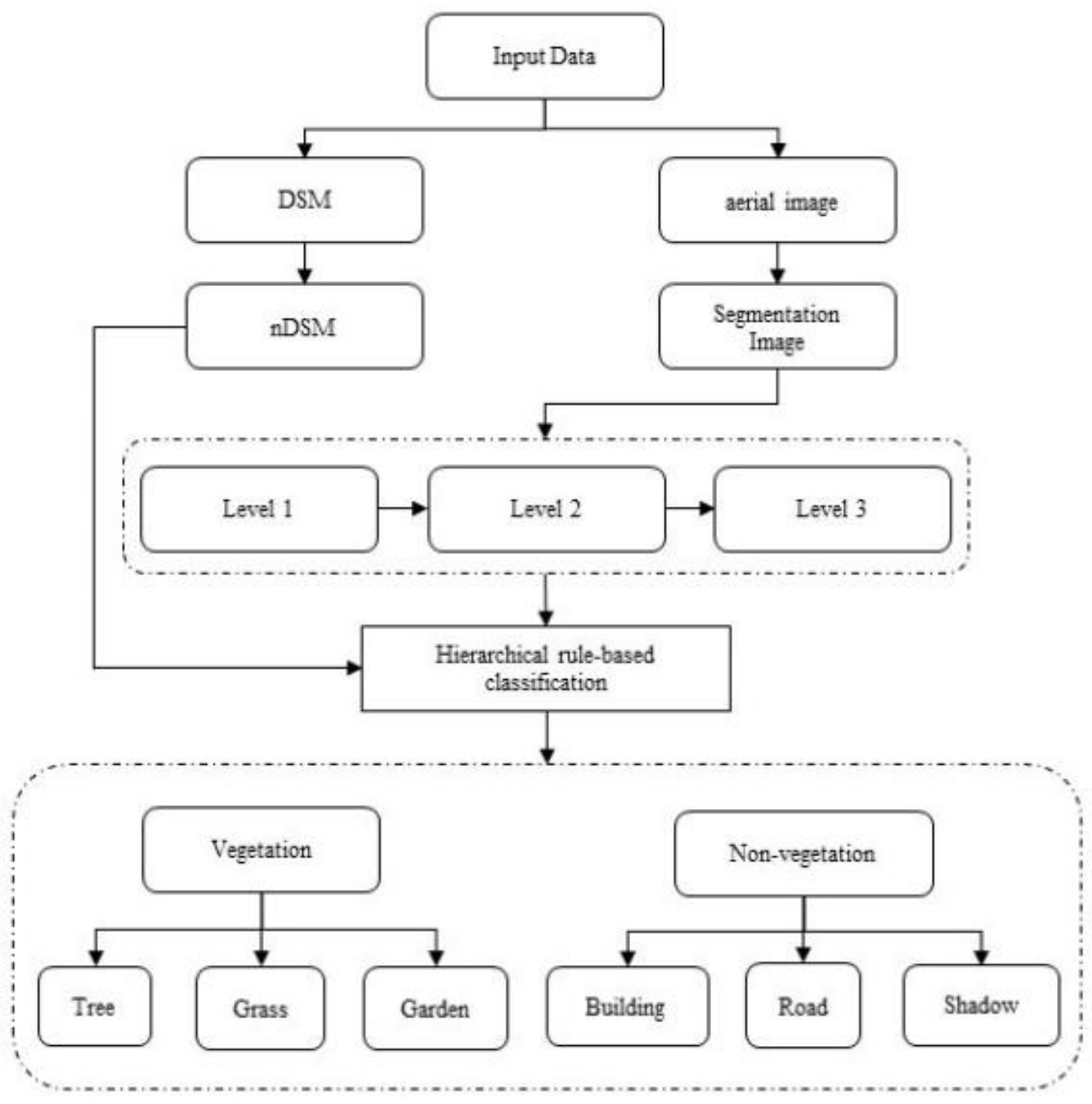

Figure 2. Flowchart of the object-based classification process 


\subsection{Image Segmentation}

Image segmentation is the process of partitioning a digital image into multiple segments (sets of pixels, also known as super pixels). Segmentation is the first step in object-based image analysis as well as the most important process in this study because its accuracy affects the overall result.(Anders et al., 2011, Kim et al., 2011). A multiresolution segmentation algorithm embedded in eCognition software(Definiens, 2013) was firstly used. The multiresolution segmentation algorithm is an optimization procedure which, for a given number of image objects, minimizes the average heterogeneity and maximizes their respective homogeneity. In the eCognition ${ }^{\circledR}$ software, segmentation performance is jointly controlled by the userdefined scale, shape and compactness parameters. Users have to repeatedly select a set of segmentation parameters and test them through a trial-and-error process, until a satisfied segmentation result is achieved(Tong et al., 2012). This operation is a timeconsuming process, and priori knowledge is required. Instead of trial and error, we used a supervised and Fuzzy-based approach to get optimal segmentation parameters for eCognition $®($ Tong et al., 2012). This approach named Fuzzy-based Segmentation Parameter optimizer (FBSP optimizer), which calculates the optimal segmentation parameters for interested object through a training process and fuzzy logic analysis in an iteration fashion(Zhou et al., 2015). Experiments demonstrate that the approach effectively reduces the operation time.

\subsection{Image Classification}

The second step in object-based image classification is classification. Due to the high resolution of the image, detail of land use and land cover could be divided into 6 classes, which are buildings, road, tree, grass, shadow and garden/water (There is no water type in the Data 1 and no garden type in Data 2). Firstly, based on the optimal segmentation result of Data 1, we used the NDVI to divide the research area into vegetation and non-vegetation parts. Second, non-vegetation areas were classified to buildings, roads and shadow. Vegetations were also broken into to grass, trees and garden in this step. A hierarchical rule-based classifier was created to classify each object to a land cover type. In the rule-set, various spectral, textural, morphological and class-related were employed (Salehi et al., 2012).

In the object-oriented classification method, the classification hierarchy system refers to the longitudinal relationship between different segmentation level and the horizonal relationship among the same level. The classification hierarchy system can be helpful for building the relation between image objects in different segmentation level. Good classification system is conductive to selecting appropriate features or combinations of feature, in which different levels can convey their characteristics description so that each level can not only use the current level's feature information, but also can use of the feature information of the adjacent level.

\begin{tabular}{lll}
\hline Class & Segmentation Level & Feature \\
\hline Vegetation & Level 1 & NDVI \\
Tree, Grass & Level 1 & nDSM \\
Building & Level 2 & Brightness, nDSM, Existence of vegetable \\
Road & Level 2 & Brightness, Area, Classified as building, Existence of vegetation, NDVI \\
Shadow & Level 2 & Brightness, NDVI \\
Garden & Level 3 & Area, GLCM Contrast, Rectangular Fit, Texture \\
\hline
\end{tabular}

Table 3. Object's features used in the rule-set hierarchy for different classes

\section{RESULTS AND DISCUSSION}

\subsection{Segmentation results}

After several experiments and the training of FBSP optimizer, the appropriate segmentation parameters were determined for two sets of data (Table 4). The visual results of two sets of data are depicted in Figure 5. The segmentation level number and three segmentation parameters (scale, shape, compactness) are different for Data 1 and Data 2 image. At level 1, the first segmentation result of image, different types of objects were separated with a small scale and shape parameters, given in Table 4. Since objects in lower levels was over-segmented, the lower levels (i.e., level 1) preferred for classifying land covers in which the spectral features are employed (i.e., vegetation)(Salehi et al., 2012). Comparing to the lower levels, higher levels such level 2 and level 3 are more suitable for the classification of larger objects. Because it's obviously that, at the higher levels, the morphological features such as shape and area and the texture feature are more important than spectral features for the classification of larger objects(Salehi et al., 2012).

\begin{tabular}{llllll}
\hline & Level & Scale & Shape & Compactness & Class \\
\hline \multirow{3}{*}{ Data 1} & 1 & 12 & 0.1 & 0.9 & Grass, Tree \\
& 2 & 57.4394 & 0.3901 & 0.8129 & Road, Building, Shadow \\
& 3 & 204.3351 & 0.3869 & 0.8063 & Garden \\
\hline \multirow{3}{*}{ Data 2} & 1 & 10 & 0.1 & 0.5 & \\
& 2 & 58.52 & 0.468 & 0.7202 & Grass, Tree \\
& 3 & 89.562 & 0.1 & 0.5 & Building, Shadow, Road \\
& 4 & 330 & 0.6703 & 0.8202 & Water \\
\hline
\end{tabular}

Table 4. Multiresolution segmentation parameters for the two images 


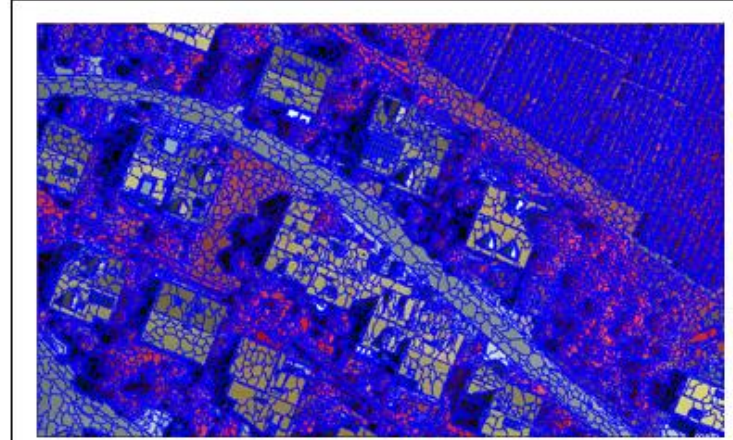

Data 1-Level1

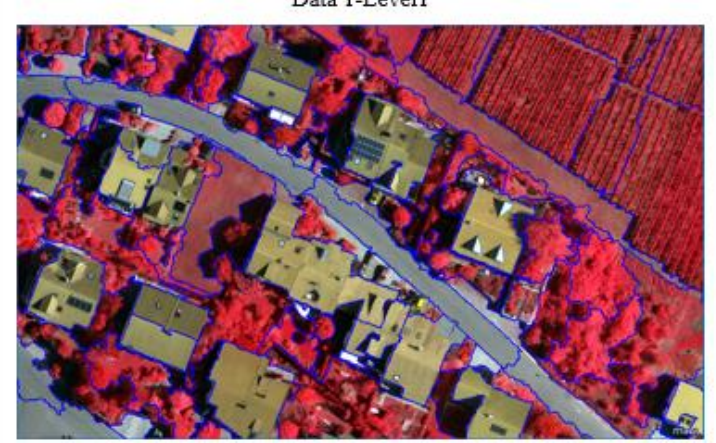

Data 1-Level3

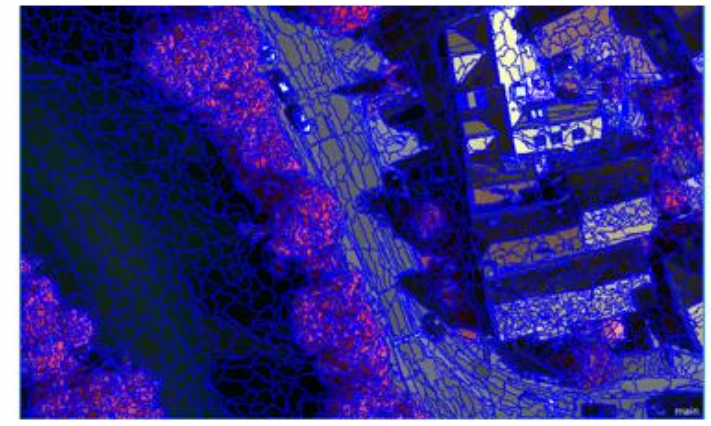

Data 2-Level1

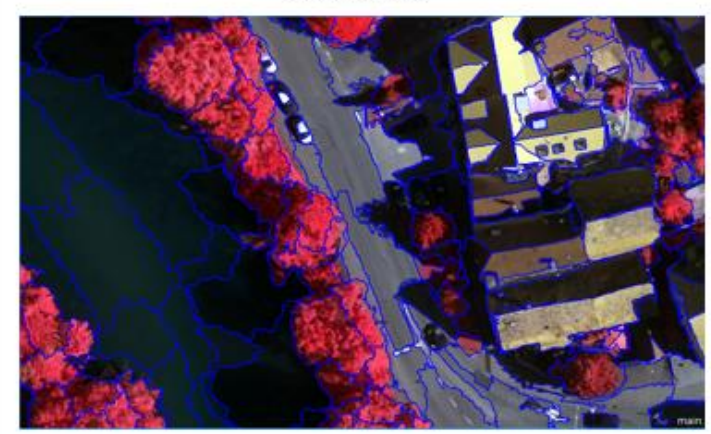

Data 2-Leve13

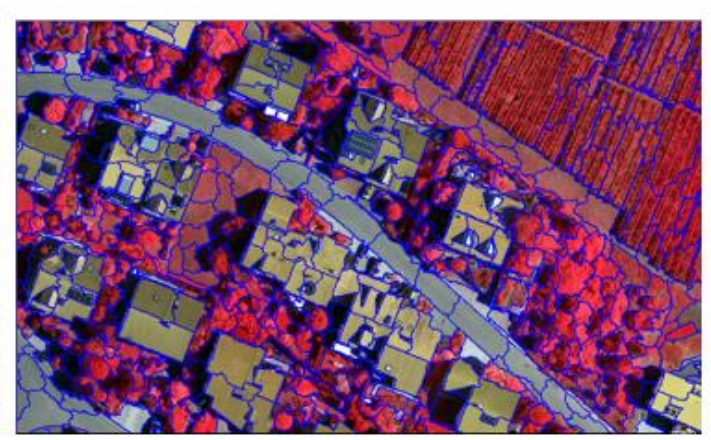

Data 1-Level2

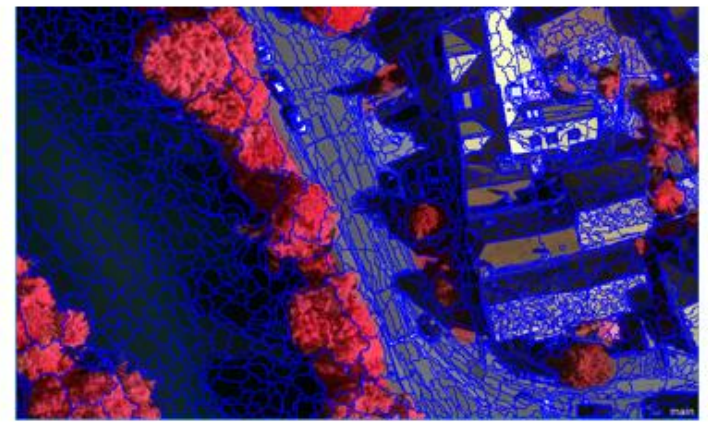

Data 2-Level2

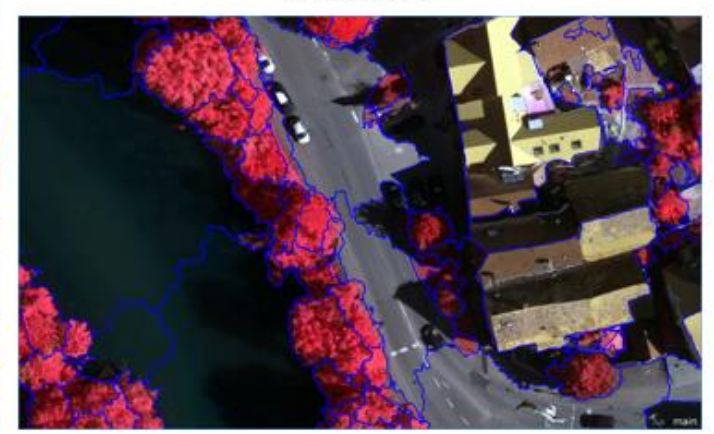

Data 2-Level4

Figure 5. Multiresolution segmentation results of Level 1, Level 2, Level 3, and Level 4 for Data 1 and Data 2 images

\subsection{Classification results}

In this step, the developed method was applied to the high resolution optical and LiDAR data that were acquired at the same time. The classification hierarchy system of Data 1 is showed in Table 7 and the classification results of two images are showed in Figure 6. The image objects were classified into trees and grass in level 1. At level 1, most of the vegetation could be well distinguished by using spectral information and height information provided by DSM data. However, some tree areas in two images were extracted as grass because they were not segmented optimally and the objects' height deriving from DSM has some deviation. At level 2, spatially adjacent segments assigned to the same group in level 1 were merged to form more meaningful landscape entities(Zhou et al., 2008). Therefore, simple features can be used to distinguish buildings, roads and shadows showed in Table 7. At level 3, the garden type was classified utilizing area, texture and shape information. In addition, the Data 2 was classified by the similar rule-set. 


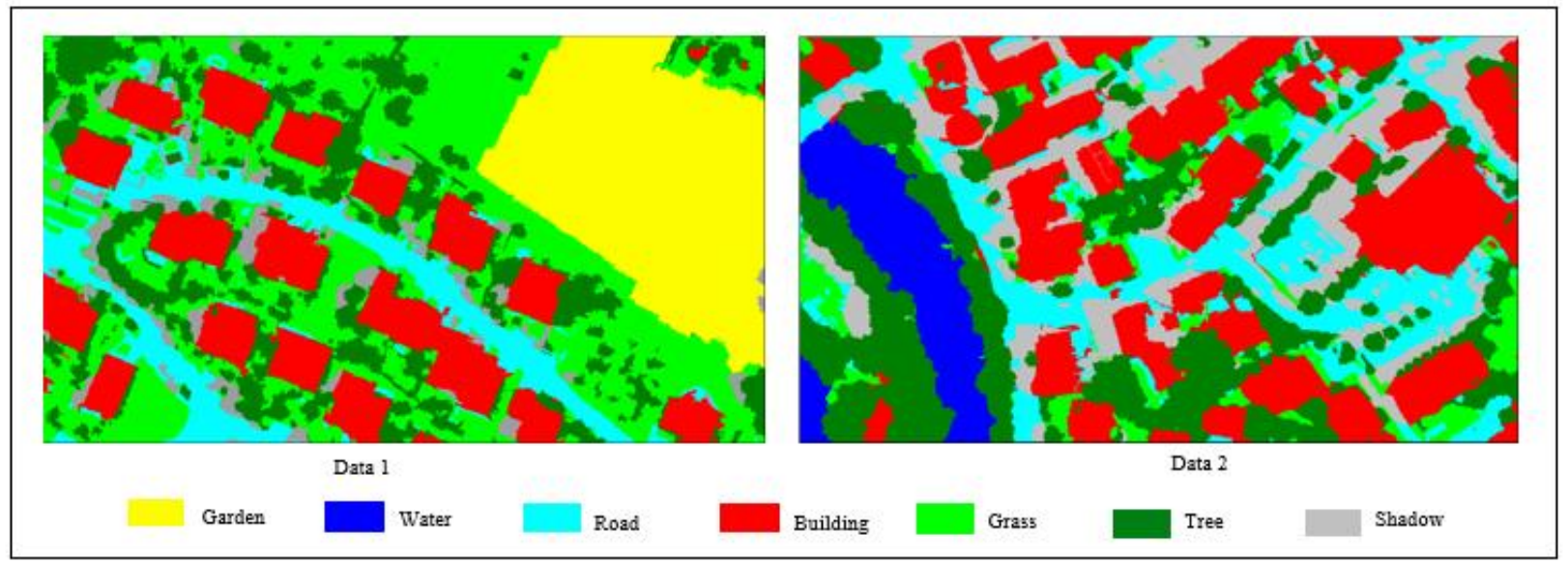

Figure 6. Classification results for the two images

\begin{tabular}{|c|c|c|c|}
\hline & Class & Features & Values \\
\hline \multirow[t]{10}{*}{ Vegetation } & Garden & Area & $>10000$ \\
\hline & & GLCM Contrast (0) & $>192$ \\
\hline & & Rectangular Fit & $>0.65$ \\
\hline & & Texture & $>17$ \\
\hline & & Existence of sub objects & Building $(1)=0 \& \operatorname{Road}(1)=0 \&$ Shadow $(1)=0$ \\
\hline & Tree & Mean nDSM & $>0.5$ \\
\hline & & Existence of super objects & Building $(1)=0 \& \operatorname{Road}(1)=0 \&$ Shadow $(1)=0 \&$ Garden $(2)=0$ \\
\hline & Grass & Mean nDSM & $<=0.5$ \\
\hline & & Existence of super objects & Building $(1)=0 \& \operatorname{Road}(1)=0 \&$ Shadow $(1)=0 \&$ Garden $(2)=0$ \\
\hline & & Classified as & Tree $=0$ \\
\hline \multirow{11}{*}{ Non-vegetation } & Building & Brightness & $>111$ \\
\hline & & Existence of sub objects & Vegetation $(1)=0$ \\
\hline & & Mean nDSM & $>0.78$ \\
\hline & Road & Area & $\neq 34$ \\
\hline & & Brightness & $>165$ \\
\hline & & Classified as & Building $=0$ \\
\hline & & Existence of sub objects & Vegetation $(1)=0$ \\
\hline & & NDVI & $<0.142$ \\
\hline & Shadow & Brightness & $<148$ \\
\hline & & NDVI & $<0.2$ \\
\hline & & Classified as & Building $=0 \&$ Road $=0$ \\
\hline
\end{tabular}

Table 7. Classification hierarchy system of Data 1

\subsection{Accuracy assessment}

In the last step, the object classification results are evaluated by using the confusion matrix including overall accuracy and Kappa coefficients. And the reference data is a total number of 400 random points that generated within the scope of each image. The confusion matrix of the accuracy assessment for Data 1 is listed in table 8 , with user's and producer's accuracy were calculated. The overall accuracy of the Data 1 classification was $85.25 \%$ and the overall kappa coefficients was 0.8171 . Among them, building, grass, shadow and garden were classified with producer accuracies of more than $91 \%$, and building, tree and garden were classified with producer accuracies of more than $94 \%$. However, the producer's accuracy of tree and the user's accuracy of grass are less $70 \%$. In other words, the same accuracy assessment using the same sampling procedure was also performed for the Data 2 (Table 9). And an overall accuracy of $89.75 \%$ and an overall kappa coefficient of 0.8684 were achieved for Data 2 image. Its value is higher than the value of Data 1. Especially, the producer's accuracies for water, tree and shadow are $100 \%, 94.06 \%$ and $93.33 \%$, respectively. In a summary, the result shows that this approach obtained a good performance.

\begin{tabular}{|c|c|c|c|c|c|c|c|c|c|}
\hline & \multicolumn{7}{|l|}{ Reference } & \multirow{2}{*}{$\begin{array}{l}\text { Producer's } \\
\text { accuracy }(\%)\end{array}$} & \multirow{2}{*}{$\begin{array}{l}\text { User's accuracy } \\
(\%)\end{array}$} \\
\hline & Building & Road & Tree & Grass & Shadow & Garden & Total & & \\
\hline Building & 69 & 2 & 0 & 0 & 0 & 0 & 71 & 95.83 & 97.18 \\
\hline Road & 2 & 25 & 0 & 3 & 0 & 0 & 30 & 73.53 & 83.33 \\
\hline Tree & 1 & 0 & 80 & 3 & 0 & 1 & 85 & 67.23 & 94.12 \\
\hline Grass & 0 & 3 & 37 & 76 & 0 & 0 & 116 & 91.57 & 65.51 \\
\hline Shadow & 0 & 4 & 2 & 0 & 27 & 0 & 33 & 100.00 & 81.82 \\
\hline Garden & 0 & 0 & 0 & 1 & 0 & 64 & 65 & 98.46 & 98.46 \\
\hline Total & 72 & 34 & 119 & 83 & 27 & 65 & 400 & & \\
\hline \multicolumn{10}{|c|}{ Overall accuracy $=85.25 \%$, Overall kappa coefficient $=0.8171$} \\
\hline
\end{tabular}

Table 8. Confusion matrix for Data 1 
The International Archives of the Photogrammetry, Remote Sensing and Spatial Information Sciences, Volume XLII-3, 2018

ISPRS TC III Mid-term Symposium "Developments, Technologies and Applications in Remote Sensing”, 7-10 May, Beijing, China

\begin{tabular}{|c|c|c|c|c|c|c|c|c|c|}
\hline \multirow[t]{2}{*}{ Classified } & \multicolumn{7}{|l|}{ Reference } & \multirow{2}{*}{$\begin{array}{l}\text { Producer's } \\
\text { accuracy }(\%)\end{array}$} & \multirow{2}{*}{$\begin{array}{l}\text { User's accuracy } \\
(\%)\end{array}$} \\
\hline & Building & Road & Tree & Grass & Shadow & Water & Total & & \\
\hline Building & 123 & 3 & 1 & 1 & 2 & 0 & 135 & 91.11 & 94.62 \\
\hline Road & 3 & 47 & 0 & 0 & 0 & 0 & 50 & 78.33 & 94.00 \\
\hline Tree & 3 & 1 & 95 & 6 & 0 & 0 & 105 & 94.06 & 90.47 \\
\hline Grass & 1 & 5 & 4 & 18 & 1 & 0 & 29 & 72.00 & 62.07 \\
\hline Shadow & 5 & 4 & 0 & 0 & 42 & 0 & 51 & 93.33 & 82.35 \\
\hline Water & 0 & 0 & 1 & 0 & 0 & 34 & 35 & 100.00 & 97.14 \\
\hline Total & 135 & 60 & 101 & 25 & 45 & 34 & 400 & & \\
\hline \multicolumn{10}{|c|}{ Overall accuracy $=89.75 \%$, Overall kappa coefficient $=0.8684$} \\
\hline
\end{tabular}

Table 9. Confusion matrix for Data 2

\section{CONCLUSION}

Based on LiDAR data and high spatial resolution optical data, the classification of land cover types was achieved in the two sets of data. After the accuracy assessment the classification results of Data 1 and Data 2, the comparison results displayed that Data 2's overall accuracy and overall kappa coefficient are a little higher than Data 1's statistical results. Therefore, it is demonstrated that this classification hierarchy system has a great potential for image classification of other areas of the same high spatial resolution image.

Although relatively good classification results were obtained through the object-oriented approach, there are two main points need to be further addressed. Firstly, the segmentation method needs to be improved to better separate different land cover types. For example, a segment contains two different ground objects in higher levels, especially the objects covered with shadows resulting in unobvious boundary of different land cover types. Secondly, a more suitable feature set for classifying also needed. For instance, the road and grass in the shadow were the main reasons for misclassification. In the future, we will focus on the method of an optimal segmentation and the extraction of a more accurate land cover classification under shadows.

\section{REFERENCE}

Anders, N. S., Seijmonsbergen, A. C.,Bouten, W. 2011. Segmentation optimization and stratified object-based analysis for semi-automated geomorphological mapping. Remote Sensing of Environment, 115(12), pp. 2976-2985.

Definiens, I. 2013. eCognition Developer 8.9 Reference Book, Place, pp. 449.

Kim, Y., Han, Y., Byun, Y., Choi, J., Han, D.,Kim, Y. 2011. Object-Based Classification of an Urban Area Through a Combination of Aerial Image and Airborne Lidar Data. ASPRS, Milwaukee.

Salehi, B., Zhang, Y., Zhong, M.,Dey, V. 2012. Object-Based Classification of Urban Areas Using VHR Imagery and Height Points Ancillary Data. Remote Sensing, 4(8), pp. 2256-2276.

Tong, H., Maxwell, T., Zhang, Y.,Dey, V. 2012. A Supervised and Fuzzy-based Approach to Determine Optimal Multiresolution Image Segmentation Parameters. Photogrammetric Engineering \& Remote Sensing, 78(10), pp. 1029-1044.

Zhou, W.,Troy, A. 2008. An object-oriented approach for analysing and characterizing urban landscape at the parcel level. International Journal of Remote Sensing, 29(11), pp. 3119-3135.

Zhou, Y.,Qiu, F. 2015. Fusion of high spatial resolution WorldView-2 imagery and LiDAR pseudo-waveform for objectbased image analysis. ISPRS Journal of Photogrammetry \& Remote Sensing, 101, pp. 221-232. 